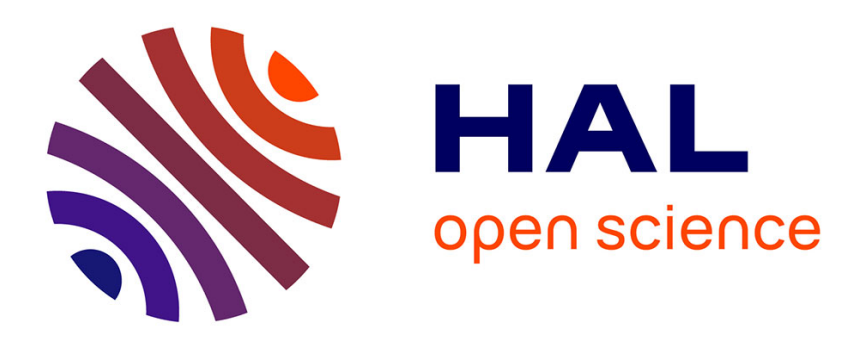

\title{
A variational inequality and applications to quasistatic problems with Coulomb friction
}

Marius Cocou

\section{To cite this version:}

Marius Cocou. A variational inequality and applications to quasistatic problems with Coulomb friction. Applicable Analysis, 2018, 97 (8), pp.1357-1371. 10.1080/00036811.2017.1376249 . hal01775320

\author{
HAL Id: hal-01775320 \\ https://hal.science/hal-01775320
}

Submitted on 24 Apr 2018

HAL is a multi-disciplinary open access archive for the deposit and dissemination of scientific research documents, whether they are published or not. The documents may come from teaching and research institutions in France or abroad, or from public or private research centers.
L'archive ouverte pluridisciplinaire HAL, est destinée au dépôt et à la diffusion de documents scientifiques de niveau recherche, publiés ou non, émanant des établissements d'enseignement et de recherche français ou étrangers, des laboratoires publics ou privés. 


\title{
A variational inequality and applications to quasistatic problems with Coulomb friction
}

\author{
Marius Cocou 1 \\ Aix-Marseille Univ, CNRS, Centrale Marseille, LMA UMR 7031, France
}

Keywords

Evolution inequalities, existence results, quasistatic problems, nonlinear materials, Coulomb friction

MSC(2010): 35Q74; 49J40; 74D10; 74H20.

\begin{abstract}
The aim of this paper is to study an evolution variational inequality that generalizes some contact problems with Coulomb friction in small deformation elasticity. Using an incremental procedure, appropriate estimates and convergence properties of the discrete solutions, the existence of a continuous solution is proved. This abstract result is applied to quasistatic contact problems with a local Coulomb friction law for nonlinear Hencky and also for linearly elastic materials.
\end{abstract}

\section{Introduction}

This paper concerns the analysis of an evolution variational inequality that represents a generalization of some quasistatic elastic problems with pointwise Coulomb friction and relaxed unilateral contact.

Existence and approximation of solutions to the quasistatic elastic problems have been studied for various contact conditions. Based on the variational formulation proposed in [1], the quasistatic unilateral contact problems with local Coulomb friction have been studied in [2, 3, 4] and the normal compliance models have been investigated by several authors, see, e.g. [5, 6, 7] and references therein. Dynamic frictional contact problems with normal compliance laws for some viscoelastic bodies have been studied in [8, 5, 9, 10, 11].

\footnotetext{
${ }^{1}$ Corresponding author:

Marius Cocou, Laboratoire de Mécanique et d'Acoustique, 4 Impasse Nikola Tesla, CS 40006, 13453 Marseille Cedex 13, France.

Email: cocou@lma.cnrs-mrs.fr
} 
A comprehensive presentation of contact models for the quasistatic processes can be found in [12]. An unified approach, which can be applied to various quasistatic problems, including unilateral and bilateral contact with nonlocal friction, or normal compliance conditions, has been considered in [13, and different (quasi)static contact problems with nonlocal friction are analyzed in [14.

A static contact problem with relaxed unilateral conditions and pointwise Coulomb friction was studied in [15], based on abstract formulations and $\mathrm{Ky}$ Fan's fixed point theorem. Recently, an extension of these results to dynamic contact problems in viscoelasticity was treated in [16, 17].

This paper extends the results presented in [15] to a new evolution variational inequality involving a nonlinear operator and with applications to two-field formulations of some nonsmooth elastic quasistatic contact problems with friction.

The paper is organized as follows. In Section 2, a general evolution variational inequality is analyzed by an incremental method. Using the Ky Fan's theorem, the existence of incremental solutions is proved. Then several estimates and compactness arguments enable to pass to limits in order to establish the existence of a continuous solution. In Section 3, applications to quasistatic contact problems with local Coulomb friction, for nonlinear Hencky and linearly elastic bodies, are presented.

\section{An implicit variational inequality}

For simplicity and also in view of applications to contact mechanics, we shall confine attention to the case when $\Omega$ is an open, bounded, connected set $\Omega \subset \mathbb{R}^{d}, d=2,3$, with the boundary $\Gamma \in C^{1,1}$ and with $\Xi$ an open part of $\Gamma$.

We denote $\Xi_{T}:=\Xi \times(0, T)$, where $0<T<+\infty$, and define the closed convex cones $L_{-}^{2}(\Xi), L_{-}^{2}\left(\Xi_{T}\right)$ in the Hilbert spaces $L^{2}(\Xi), L^{2}\left(\Xi_{T}\right)$, respectively, as follows:

$$
\begin{aligned}
& L_{-}^{2}(\Xi):=\left\{\delta \in L^{2}(\Xi) ; \delta \leq 0 \text { a.e. in } \Xi\right\}, \\
& L_{-}^{2}\left(\Xi_{T}\right):=\left\{\delta \in L^{2}\left(0, T ; L^{2}(\Xi)\right) ; \delta \leq 0 \text { a.e. in } \Xi_{T}\right\} .
\end{aligned}
$$

Let $\underline{\kappa}, \bar{\kappa}: \mathbb{R} \rightarrow \mathbb{R}$ be two mappings with $\underline{\kappa}$ lower semicontinuous and $\bar{\kappa}$ upper semicontinuous, satisfying the following conditions:

$$
\begin{aligned}
& \underline{\kappa}(s) \leq \bar{\kappa}(s) \leq 0 \quad \forall s \in \mathbb{R}, \\
& \exists r_{0} \geq 0 \text { such that }|\underline{\kappa}(s)| \leq r_{0} \forall s \in \mathbb{R} .
\end{aligned}
$$


For every $\zeta \in L^{2}(\Xi)$, define the following subset of $L_{-}^{2}(\Xi)$ :

$$
\Lambda(\zeta)=\left\{\eta \in L_{-}^{2}(\Xi) ; \underline{\kappa} \circ \zeta \leq \eta \leq \bar{\kappa} \circ \zeta \text { a.e. in } \Xi\right\},
$$

which is clearly nonempty, because the bounding functions belong to the respective set, closed and convex.

Since meas $(\Xi)<\infty$ and $\underline{\kappa}, \bar{\kappa}$ satisfy $(2)$, it is also readily seen that for all $\zeta \in L^{2}(\Xi)$ the set $\Lambda(\zeta)$ is bounded in norm in $L^{2}(\Xi)$ by $R_{0}=r_{0}(\text { meas }(\Xi))^{1 / 2}$ and in $L^{\infty}(\Xi)$ by $r_{0}$.

The following compactness theorem proved in [18] will be used in this paper.

Theorem 2.1. Let $\hat{X}, \hat{U}$ and $\hat{Y}$ be three Banach spaces such that $\hat{X} \subset \hat{U} \subset \hat{Y}$ with compact embedding from $\hat{X}$ into $\hat{U}$.

(i) Let $\mathcal{G}$ be bounded in $L^{p}(0, T ; \hat{X})$, where $1 \leq p<\infty$, and $\partial \mathcal{G} / \partial t:=$ $\{\dot{f} ; f \in \mathcal{G}\}$ be bounded in $L^{1}(0, T ; \hat{Y})$. Then $\mathcal{G}$ is relatively compact in $L^{p}(0, T ; \hat{U})$.

(ii) Let $\mathcal{G}$ be bounded in $L^{\infty}(0, T ; \hat{X})$ and $\partial \mathcal{G} / \partial t$ be bounded in $L^{r}(0, T ; \hat{Y})$, where $r>1$. Then $\mathcal{G}$ is relatively compact in $C([0, T] ; \hat{U})$.

If $H$ is a Hilbert space, unless otherwise stated we shall denote by $\langle., .\rangle_{H}$ its inner product and by $\|\cdot\|_{H}$ the corresponding norm.

Let $(V,\|\|,.\langle.,\rangle$.$) and \left(U,\|.\|_{U}\right)$ be two Hilbert spaces such that $V \subset U$ with continuous and compact embedding.

Consider a functional $F: V \rightarrow \mathbb{R}$ differentiable on $V$ and assume that its derivative $F^{\prime}: V \rightarrow V$ is strongly monotone and Lipschitz continuous, that is there exist two constants $\alpha, \beta>0$ for which

$$
\alpha\|v-u\|^{2} \leq\left\langle F^{\prime}(v)-F^{\prime}(u), v-u\right\rangle
$$

and

$$
\left\|F^{\prime}(v)-F^{\prime}(u)\right\| \leq \beta\|v-u\|
$$

for all $u, v \in V$.

Using the relations

$$
\begin{aligned}
F(v) & -F(u)=\int_{0}^{1}\left\langle F^{\prime}(u+r(v-u)), v-u\right\rangle d r \\
& =\left\langle F^{\prime}(u), v-u\right\rangle+\int_{0}^{1}\left\langle F^{\prime}(u+r(v-u))-F^{\prime}(u), v-u\right\rangle d r
\end{aligned}
$$


and (4), (5), it is easily seen that for all $u, v \in V$ it results

$$
\begin{gathered}
\left\langle F^{\prime}(u), v-u\right\rangle+\frac{\alpha}{2}\|v-u\|^{2} \leq F(v)-F(u) \\
\leq\left\langle F^{\prime}(u), v-u\right\rangle+\frac{\beta}{2}\|v-u\|^{2} .
\end{gathered}
$$

We remark that since $F$ satisfies (6), it follows that $F$ is strictly convex and sequentially weakly lower semicontinuous on $V$.

Let $\left(X,\|\cdot\|_{X}\right)$ be a Hilbert space such that $X \subset L^{2}(\Gamma)$ with continuous and compact embedding, and $l_{0}: V \rightarrow X, l: V \rightarrow L^{2}(\Xi), \phi: L_{-}^{2}(\Xi) \times V \rightarrow$ $\mathbb{R}$ be three mappings satisfying the following conditions:

$l_{0}$ is linear and continuous,

$\exists k_{1}>0$ such that $\forall v_{1}, v_{2} \in V$,

$\left\|l\left(v_{1}\right)-l\left(v_{2}\right)\right\|_{L^{2}(\Xi)} \leq k_{1}\left\|v_{1}-v_{2}\right\|_{U}$,

$\forall \gamma, \delta \in L_{-}^{2}(\Xi), \forall v, w \in V$ verifying $\gamma \in \Lambda(l(v))$ and $\delta \in \Lambda(l(w))$, $\left\langle\gamma-\delta, l_{0}(v-w)\right\rangle_{L^{2}(\Xi)} \leq 0$.

$\forall \gamma \in L_{-}^{2}(\Xi), \forall \theta \geq 0, \forall v_{1}, v_{2}, v \in V$,

$\phi\left(\gamma, v_{1}+v_{2}\right) \leq \phi\left(\gamma, v_{1}\right)+\phi\left(\gamma, v_{2}\right)$,

$\phi(\gamma, \theta v)=\theta \phi(\gamma, v)$,

$\forall v \in V, \quad \phi(0, v)=0$,

$\exists k_{2}, k_{3}>0$ such that $\forall \gamma, \delta \in L_{-}^{2}(\Xi), \forall v \in V$,

$|\phi(\gamma, v)-\phi(\delta, v)| \leq k_{2}\|\gamma-\delta\|_{L^{2}(\Xi)}\|v\|_{U}$,

$|\phi(\gamma, v)-\phi(\delta, v)| \leq k_{3}\|\gamma-\delta\|_{X^{\prime}}\|v\|$,

$\exists k_{4}>0$ such that $\left\|\gamma_{1}-\gamma_{2}\right\|_{X^{\prime}} \leq k_{4}\left(\left\|u_{1}-u_{2}\right\|+\left\|f_{1}-f_{2}\right\|\right)$,

for all $\gamma_{1,2} \in L_{-}^{2}(\Xi), u_{1,2}, f_{1,2}, d_{1,2} \in V$ verifying

$\left(Q_{1}\right)\left\langle F^{\prime}\left(u_{1}\right), v-u_{1}\right\rangle-\left\langle\gamma_{1}, l_{0}\left(v-u_{1}\right)\right\rangle_{L^{2}(\Xi)}$

$$
+\phi\left(\gamma_{1}, v-d_{1}\right)-\phi\left(\gamma_{1}, u_{1}-d_{1}\right) \geq\left\langle f_{1}, v-u_{1}\right\rangle \quad \forall v \in V,
$$

$\left(Q_{2}\right)\left\langle F^{\prime}\left(u_{2}\right), v-u_{2}\right\rangle-\left\langle\gamma_{2}, l_{0}\left(v-u_{2}\right)\right\rangle_{L^{2}(\Xi)}$

$$
+\phi\left(\gamma_{2}, v-d_{2}\right)-\phi\left(\gamma_{2}, u_{2}-d_{2}\right) \geq\left\langle f_{2}, v-u_{2}\right\rangle \quad \forall v \in V,
$$

and we assume that $k_{3} k_{4}<\alpha$. 
Let $f \in W^{1,2}(0, T ; V), u^{0} \in V, \lambda^{0} \in \Lambda\left(l\left(u^{0}\right)\right)$ be given and satisfy the following compatibility condition:

$$
\begin{aligned}
& \left\langle F^{\prime}\left(u^{0}\right), v-u^{0}\right\rangle-\left\langle\lambda^{0}, l_{0}\left(v-u^{0}\right)\right\rangle_{L^{2}(\Xi)} \\
& \quad+\phi\left(\lambda^{0}, v\right)-\phi\left(\lambda^{0}, u^{0}\right) \geq\left\langle f(0), v-u^{0}\right\rangle \quad \forall v \in V .
\end{aligned}
$$

Consider the following problem.

Problem $Q$ : Find $u \in W^{1,2}(0, T ; V), \lambda \in W^{1,2}\left(0, T ; X^{\prime}\right)$ such that $u(0)=$ $u^{0}, \lambda(0)=\lambda^{0}, \lambda(t) \in \Lambda(l(u(t)))$ for almost all $t \in(0, T)$, and

$$
\begin{aligned}
& \left\langle F^{\prime}(u), v-\dot{u}\right\rangle-\left\langle\lambda, l_{0}(v-\dot{u})\right\rangle_{L^{2}(\Xi)}+\phi(\lambda, v) \\
& -\phi(\lambda, \dot{u}) \geq\langle f, v-\dot{u}\rangle \quad \forall v \in V \quad \text { a.e. on }(0, T) .
\end{aligned}
$$

\subsection{Incremental formulations}

For $n \in N^{*}$, we set $\Delta t:=T / n, t_{i}:=i \Delta t, i=0,1, \ldots, n$. If $\theta$ is a continuous function of $t \in[0, T]$ valued in some vector space, we use the notations $\theta^{i}:=\theta\left(t_{i}\right)$ unless $\theta=u$, and if $\varpi^{i}, \forall i \in\{0,1, \ldots, n\}$, are elements of some vector space, then we set

$$
\partial \varpi^{i}:=\frac{\varpi^{i+1}-\varpi^{i}}{\Delta t}, \quad \Delta \varpi^{i}:=\varpi^{i+1}-\varpi^{i} \quad \forall i \in\{0,1, \ldots, n-1\} .
$$

We approximate the problem $Q$ using the following sequence of incremental problems $\left(Q^{i, n}\right)_{i=0,1, \ldots, n-1}$.

Problem $\boldsymbol{Q}^{i, n}$ : Find $u^{i+1} \in V, \lambda^{i+1} \in \Lambda\left(l\left(u^{i+1}\right)\right)$ such that

$$
\begin{array}{r}
\left\langle F^{\prime}\left(u^{i+1}\right), v-\partial u^{i}\right\rangle-\left\langle\lambda^{i+1}, l_{0}\left(v-\partial u^{i}\right)\right\rangle_{L^{2}(\Xi)}+\phi\left(\lambda^{i+1}, v\right) \\
-\phi\left(\lambda^{i+1}, \partial u^{i}\right) \geq\left\langle f^{i+1}, v-\partial u^{i}\right\rangle \quad \forall v \in V .
\end{array}
$$

It is easily seen that for all $i \in\{0,1, \ldots, n-1\}$ the problem $Q^{i, n}$ is equivalent to the following implicit variational inequality.

Problem $\hat{\boldsymbol{Q}}^{i, n}$ : Find $u^{i+1} \in V, \lambda^{i+1} \in \Lambda\left(l\left(u^{i+1}\right)\right)$ such that

$$
\begin{gathered}
\left\langle F^{\prime}\left(u^{i+1}\right), v-u^{i+1}\right\rangle-\left\langle\lambda^{i+1}, l_{0}\left(v-u^{i+1}\right)\right\rangle_{L^{2}(\Xi)}+\phi\left(\lambda^{i+1}, v-u^{i}\right) \\
-\phi\left(\lambda^{i+1}, u^{i+1}-u^{i}\right) \geq\left\langle f^{i+1}, v-u^{i+1}\right\rangle \quad \forall v \in V .
\end{gathered}
$$


Let us define the following functions:

$$
\begin{aligned}
& u_{n}(0)=\hat{u}_{n}(0)=u^{0}, \lambda_{n}(0)=\lambda^{0}, f_{n}(0)=f^{0} \text { and } \\
& \forall i \in\{0,1, \ldots, n-1\}, \forall t \in\left(t_{i}, t_{i+1}\right], \\
& u_{n}(t)=u^{i+1}, \lambda_{n}(t)=\lambda^{i+1}, \\
& \hat{u}_{n}(t)=u^{i}+\left(t-t_{i}\right) \partial u^{i}, \\
& \hat{\lambda}_{n}(t)=\lambda^{i}+\left(t-t_{i}\right) \partial \lambda^{i}, \\
& f_{n}(t)=f^{i+1} .
\end{aligned}
$$

Then for all $n \in N^{*}$ each of the sequences of inequalities $\left(Q^{i, n}\right)_{i=0,1, \ldots, n-1}$, $\left(\hat{Q}^{i, n}\right)_{i=0,1, \ldots, n-1}$ is equivalent to the following incremental formulation.

Problem $\boldsymbol{Q}^{n}$ : Find $u_{n} \in L^{2}(0, T ; V), \lambda_{n} \in L^{2}\left(\Xi_{T}\right)$ such that $\lambda_{n}(t) \in$ $\Lambda\left(l\left(u_{n}(t)\right)\right) \forall t \in(0, T)$ and

$$
\begin{array}{r}
\left\langle F^{\prime}\left(u_{n}(t)\right), v-\frac{d}{d t} \hat{u}_{n}(t)\right\rangle-\left\langle\lambda_{n}(t), l_{0}\left(v-\frac{d}{d t} \hat{u}_{n}(t)\right)\right\rangle_{L^{2}(\Xi)} \\
+\phi\left(\lambda_{n}(t), v\right)-\phi\left(\lambda_{n}(t), \frac{d}{d t} \hat{u}_{n}(t)\right) \\
\geq\left\langle f_{n}(t), v-\frac{d}{d t} \hat{u}_{n}(t)\right\rangle \quad \forall v \in V, \text { a.e. on }(0, T) .
\end{array}
$$

First, we prove the existence of a solution to the incremental problem $\hat{Q}^{i, n}$ by a fixed point method.

Let $\Phi^{i}: L_{-}^{2}(\Xi) \rightarrow 2^{L_{-}^{2}(\Xi)} \backslash\{\emptyset\}$ be the set-valued mapping defined by

$$
\text { for all } \gamma \in L_{-}^{2}(\Xi) \quad \Phi^{i}(\gamma)=\Lambda\left(l\left(u_{\gamma}\right)\right)
$$

where $u_{\gamma}$ is the solution of the following variational inequality of the second kind: find $u_{\gamma} \in V$ such that

$$
\begin{gathered}
\left\langle F^{\prime}\left(u_{\gamma}\right), v-u_{\gamma}\right\rangle-\left\langle\gamma, l_{0}\left(v-u_{\gamma}\right)\right\rangle_{L^{2}(\Xi)}+\phi\left(\gamma, v-u^{i}\right) \\
-\phi\left(\gamma, u_{\gamma}-u^{i}\right) \geq\left\langle f^{i+1}, v-u_{\gamma}\right\rangle \quad \forall v \in V .
\end{gathered}
$$

It is easily seen that $\lambda$ is a fixed point of $\Phi^{i}$, i.e. $\lambda \in \Phi^{i}(\lambda)$, iff $\left(u^{i+1}, \lambda^{i+1}\right)=$ $\left(u_{\lambda}, \lambda\right)$ is a solution of the problem $\hat{Q}^{i, n}$.

We shall prove the existence of a fixed point of the multifunction $\Phi^{i}$ by using a corollary of the Ky Fan's fixed point theorem [19, proved in [15] in the particular case of a reflexive Banach space. 
Definition 2.1. Let $Y$ be a reflexive Banach space, $D$ a weakly closed set in $Y$, and $\Phi: D \rightarrow 2^{Y} \backslash\{\emptyset\}$ be a multivalued function. $\Phi$ is called sequentially weakly upper semicontinuous if $z_{p} \rightarrow z, y_{p} \in \Phi\left(z_{p}\right)$ and $y_{p} \rightarrow y$ imply $y \in$ $\Phi(z)$.

Proposition 2.1. ([15]) Let $Y$ be a reflexive Banach space, $D$ a convex, closed and bounded set in $Y$, and $\Phi: D \rightarrow 2^{D} \backslash\{\emptyset\}$ a sequentially weakly upper semicontinuous multivalued function such that $\Phi(z)$ is convex for every $z \in D$. Then $\Phi$ has a fixed point.

Note that since $Y$ is a reflexive Banach space and $D$ is convex, closed and bounded, there is no assumption that $Y$ is separable, see [15, 20].

Theorem 2.2. Assume that (1- 5), (7- 14) hold. Then there exists $\lambda \in$ $L_{-}^{2}(\Xi)$ such that $\lambda \in \Phi^{i}(\lambda)$ and $\left(u^{i+1}, \lambda^{i+1}\right)=\left(u_{\lambda}, \lambda\right)$ is a solution of the problem $\hat{Q}^{i, n}$.

Proof. We apply Proposition 2.1 to $\Phi=\Phi^{i}, Y=L^{2}(\Xi)$ and $D=L_{-}^{2}(\Xi) \cap$ $\left\{\zeta \in L^{2}(\Xi) ;\|\zeta\|_{L^{2}(\Xi)} \leq R_{0}\right\}$.

The set $D \subset L^{2}(\Xi)$ is clearly convex, closed and bounded.

By (4), (5), (7), (8), (10-14), for every $\gamma \in D$ the classical variational inequality (23) has a unique solution $u_{\gamma}$.

Since for each $\zeta \in L^{2}(\Xi)$ the set $\Lambda(\zeta)$ is nonempty, convex, closed, and bounded by $R_{0}$, it follows that $\Phi^{i}(\gamma)=\Lambda\left(l\left(u_{\gamma}\right)\right)$ is a nonempty, convex and closed subset of $D$ for every $\gamma \in D$.

In order to prove that the multifunction $\Phi^{i}$ is sequentially weakly upper semicontinuous, let $\gamma_{p} \rightarrow \gamma$ in $L^{2}(\Xi), \gamma_{p} \in D, \eta_{p} \in \Phi^{i}\left(\gamma_{p}\right) \forall p \in \mathbb{N}, \eta_{p} \rightarrow \eta$ in $L^{2}(\Xi)$ and let us verify that $\eta \in \Phi^{i}(\gamma)$.

Let $u_{\gamma_{p}} \in V$ be the solution of the variational inequality

$$
\begin{gathered}
\left\langle F^{\prime}\left(u_{\gamma_{p}}\right), v-u_{\gamma_{p}}\right\rangle-\left\langle\gamma_{p}, l_{0}\left(v-u_{\gamma_{p}}\right)\right\rangle_{L^{2}(\Xi)}+\phi\left(\gamma_{p}, v-u^{i}\right) \\
-\phi\left(\gamma_{p}, u_{\gamma_{p}}-u^{i}\right) \geq\left\langle f^{i+1}, v-u_{\gamma_{p}}\right\rangle \quad \forall v \in V .
\end{gathered}
$$

Taking $v=0$ in (24) and using (10), we obtain

$$
\left\langle F^{\prime}\left(u_{\gamma_{p}}\right)-F^{\prime}(0), u_{\gamma_{p}}\right\rangle \leq\left\langle\gamma_{p}, l_{0}\left(u_{\gamma_{p}}\right)\right\rangle_{L^{2}(\Xi)}+\phi\left(\gamma_{p},-u_{\gamma_{p}}\right)+\left\langle f^{i+1}-F^{\prime}(0), u_{\gamma_{p}}\right\rangle,
$$

so that, by (4), (7), (12), (14),

$$
\alpha\left\|u_{\gamma_{p}}\right\|^{2} \leq\left(\left\|\gamma_{p}\right\|_{X^{\prime}}\left\|l_{0}\right\|+k_{3}\left\|\gamma_{p}\right\|_{X^{\prime}}+\left\|f^{i+1}\right\|+\left\|F^{\prime}(0)\right\|\right)\left\|u_{\gamma_{p}}\right\|,
$$

which implies

$$
\left.\left\|u_{\gamma_{p}}\right\| \leq \alpha^{-1}\left(\left\|l_{0}\right\|+k_{3}\right)\left\|\gamma_{p}\right\|_{X^{\prime}}+\left\|f^{i+1}\right\|+\left\|F^{\prime}(0)\right\|\right) .
$$


Thus

$$
\left\|u_{\gamma_{p}}\right\| \leq C_{1}\left(\left\|\gamma_{p}\right\|_{X^{\prime}}+\left\|f^{i+1}\right\|+\left\|F^{\prime}(0)\right\|\right) \quad \forall p \in \mathbb{N},
$$

where $C_{1}=\alpha^{-1} \max \left(\left\|l_{0}\right\|+k_{3}, 1\right)$.

As the sequence $\left(\gamma_{p}\right)_{p}$ is bounded in $L^{2}(\Xi)$, it follows that $\left(u_{\gamma_{p}}\right)_{p}$ is bounded in $V$ which implies that there exists a subsequence, still denoted by $\left(u_{\gamma_{p}}\right)_{p}$, and an element $u \in V$ such that

$$
u_{\gamma_{p}} \rightarrow u \text { in } V \text {. }
$$

By (6) and (10), the inequality (24) implies

$$
\begin{gathered}
F(v)-F\left(u_{\gamma_{p}}\right)-\left\langle\gamma_{p}, l_{0}\left(v-u_{\gamma_{p}}\right)\right\rangle_{L^{2}(\Xi)}+\phi\left(\gamma_{p}, v-u_{\gamma_{p}}\right) \\
\geq\left\langle f^{i+1}, v-u_{\gamma_{p}}\right\rangle+\frac{\alpha}{2}\left\|v-u_{\gamma_{p}}\right\|^{2} \quad \forall v \in V,
\end{gathered}
$$

and taking $v=u$, we obtain

$$
\begin{gathered}
F(u)-F\left(u_{\gamma_{p}}\right)- \\
\geq\left\langle\gamma_{p}, l_{0}\left(u-u_{\gamma_{p}}\right)\right\rangle_{L^{2}(\Xi)}+\phi\left(\gamma_{p}, u-u_{\gamma_{p}}\right) \\
\geq\left\langle f^{i+1}, u-u_{\gamma_{p}}\right\rangle+\frac{\alpha}{2}\left\|u-u_{\gamma_{p}}\right\|^{2} .
\end{gathered}
$$

As $F$ is sequentially weakly lower semicontinuous, using the previous relation, (7), 13) and the compact embeddings $X \subset L^{2}(\Xi), V \subset U$, we have

$$
\begin{aligned}
\limsup _{p \rightarrow \infty} & \frac{\alpha}{2}\left\|u-u_{\gamma_{p}}\right\|^{2} \\
\leq & F(u)+\limsup _{p \rightarrow \infty}\left(-F\left(u_{\gamma_{p}}\right)\right)+\lim _{p \rightarrow \infty}\left|\left\langle\gamma_{p}, l_{0}\left(u-u_{\gamma_{p}}\right)\right\rangle_{L^{2}(\Xi)}\right| \\
& +\lim _{p \rightarrow \infty} \phi\left(\gamma_{p}, u-u_{\gamma_{p}}\right)-\lim _{p \rightarrow \infty}\left\langle f^{i+1}, u-u_{\gamma_{p}}\right\rangle \\
& \leq F(u)-\liminf _{p \rightarrow \infty} F\left(u_{\gamma_{p}}\right)+\lim _{p \rightarrow \infty}\left\|\gamma_{p}\right\|_{L^{2}(\Xi)}\left\|l_{0}\left(u-u_{\gamma_{p}}\right)\right\|_{L^{2}(\Xi)} \\
& +\lim _{p \rightarrow \infty} k_{2}\left\|\gamma_{p}\right\|_{L^{2}(\Xi)}\left\|u-u_{\gamma_{p}}\right\|_{U}-\lim _{p \rightarrow \infty}\left\langle f^{i+1}, u-u_{\gamma_{p}}\right\rangle \\
& =F(u)-\liminf _{p \rightarrow \infty} F\left(u_{\gamma_{p}}\right) \leq 0,
\end{aligned}
$$

which proves that

$$
u_{\gamma_{p}} \rightarrow u \text { in } V \text {. }
$$

Passing to the limit in (24), it follows that $u$ is a solution of (23) and since its solution is unique we obtain that $u_{\gamma}=u=\lim _{p \rightarrow \infty} u_{\gamma_{p}}$.

Now, the relation $\eta^{p} \in \Phi^{i}\left(\gamma_{p}\right)$ implies

$$
\eta^{p} \in \Lambda\left(l\left(u_{\gamma_{p}}\right)\right)
$$


that is

$$
\underline{\kappa} \circ l_{p} \leq \eta^{p} \leq \bar{\kappa} \circ l_{p} \text { a.e. in } \Xi,
$$

for all $p \in \mathbb{N}$, where $l_{p}:=l\left(u_{\gamma_{p}}\right)$. The relations $(29)$ are equivalent to

$$
\int_{\omega} \underline{\kappa} \circ l_{p} \leq \int_{\omega} \eta^{p} \leq \int_{\omega} \bar{\kappa} \circ l_{p}
$$

for every measurable subset $\omega \subset \Xi$ and for all $p \in \mathbb{N}$.

Using (28), (8), the semi-continuity of $\underline{\kappa}$ and $\bar{\kappa}$, the relations (1), (2), the convergence property $\int_{\omega} \eta^{p} \rightarrow \int_{\omega} \eta$, and passing to limits according to Fatou's lemma, we obtain

$$
\int_{\omega} \underline{\kappa} \circ l\left(u_{\gamma}\right) \leq \int_{\omega} \eta \leq \int_{\omega} \bar{\kappa} \circ l\left(u_{\gamma}\right)
$$

for every measurable subset $\omega \subset \Xi$, which implies $\eta \in \Phi^{i}(\gamma)$.

By Proposition 2.1 there exists a fixed point $\lambda$ of $\Phi^{i}$ and $\left(u_{\lambda}, \lambda\right)$ is clearly a solution to the problem $\hat{Q}^{i, n}$.

Remark 2.1. This existence result insures also that there exists $\left(u^{0}, \lambda^{0}\right)$ satisfying the compatibility condition (17).

\subsection{Existence of a solution to the continuous problem}

We now establish some useful estimates independent of $n$ for the solutions of the incremental formulations $\hat{Q}^{i, n}$ and $Q^{n}$.

Lemma 2.1. Under the above hypotheses, for all $n \in N^{*}$ and all $i \in$ $\{0,1, \ldots, n-1\}$ the following estimates hold:

$$
\begin{gathered}
\left\|u^{i+1}\right\| \leq C_{1}\left(\left\|\lambda^{i+1}\right\|_{X^{\prime}}+\left\|f^{i+1}\right\|+\left\|F^{\prime}(0)\right\|\right), \\
\left.\left\|\Delta u^{i}\right\| \leq \frac{k_{3}}{\alpha}\left\|\Delta \lambda^{i}\right\|_{X^{\prime}}+\frac{1}{\alpha}\left\|\Delta f^{i}\right\|\right), \\
\left\|\Delta \lambda^{i}\right\|_{X^{\prime}} \leq k_{4}\left(\left\|\Delta u^{i}\right\|+\left\|\Delta f^{i}\right\|\right), \\
\left\|\Delta u^{i}\right\| \leq C_{2}\left\|\Delta f^{i}\right\|, \\
\left\|\Delta \lambda^{i}\right\|_{X^{\prime}} \leq C_{3}\left\|\Delta f^{i}\right\|, \\
\text { where } C_{2}=\frac{k_{3} k_{4}+1}{\alpha-k_{3} k_{4}}, C_{3}=\frac{(\alpha+1) k_{4}}{\alpha-k_{3} k_{4}} .
\end{gathered}
$$


Proof. By similar arguments to those that enabled to prove (25), using (20) the estimate (31) follows.

If we take $v=u^{i}$ in 20 then

$$
\begin{aligned}
& \left\langle F^{\prime}\left(u^{i+1}\right), u^{i}-u^{i+1}\right\rangle-\left\langle\lambda^{i+1}, l_{0}\left(u^{i}-u^{i+1}\right)\right\rangle_{L^{2}(\Xi)} \\
& -\phi\left(\lambda^{i+1}, u^{i+1}-u^{i}\right) \geq\left\langle f^{i+1}, u^{i}-u^{i+1}\right\rangle,
\end{aligned}
$$

and taking $v=u^{i+1}$ in 20 , corresponding to $i-1$ if $i \geq 1$, or in (17) if $i=0$, we have

$$
\begin{gathered}
\left\langle F^{\prime}\left(u^{i}\right), u^{i+1}-u^{i}\right\rangle-\left\langle\lambda^{i}, l_{0}\left(u^{i+1}-u^{i}\right)\right\rangle_{L^{2}(\Xi)}+\phi\left(\lambda^{i}, u^{i+1}-u^{i-1}\right) \\
-\phi\left(\lambda^{i}, u^{i}-u^{i-1}\right) \geq\left\langle f^{i}, u^{i+1}-u^{i}\right\rangle .
\end{gathered}
$$

By (10), the inequalities (36) and (37) imply

$$
\begin{aligned}
& \left\langle F^{\prime}\left(u^{i+1}\right)-F^{\prime}\left(u^{i}\right), u^{i+1}-u^{i}\right\rangle \leq\left\langle\lambda^{i+1}-\lambda^{i}, l_{0}\left(u^{i+1}-u^{i}\right)\right\rangle_{L^{2}(\Xi)} \\
& \quad+\phi\left(\lambda^{i}, u^{i+1}-u^{i}\right)-\phi\left(\lambda^{i+1}, u^{i+1}-u^{i}\right)+\left\langle f^{i+1}-f^{i}, u^{i+1}-u^{i}\right\rangle .
\end{aligned}
$$

As $\left(u^{i+1}, \lambda^{i+1}\right)$ and $\left(u^{i}, \lambda^{i}\right)$ are solutions of $\hat{Q}^{i+1, n}$ and $\hat{Q}^{i, n}$, respectively, we have $\lambda^{i+1} \in \Lambda\left(l\left(u^{i+1}\right)\right), \quad \lambda^{i} \in \Lambda\left(l\left(u^{i}\right)\right)$, so that by $(9)$

$$
\left\langle\lambda^{i+1}-\lambda^{i}, l_{0}\left(u^{i+1}-u^{i}\right)\right\rangle_{L^{2}(\Xi)} \leq 0 .
$$

Using this relation, (4) and (14) in (38), we have

$$
\alpha\left\|u^{i+1}-u^{i}\right\|^{2} \leq k_{3}\left\|\lambda^{i+1}-\lambda^{i}\right\|_{X^{\prime}}\left\|u^{i+1}-u^{i}\right\|+\left\|f^{i+1}-f^{i}\right\|\left\|u^{i+1}-u^{i}\right\|,
$$

from which $(32)$ follows.

From $(15)$, we obtain (33) and by $(32),(33)$ the estimates $(34),(35)$ can be easily verified.

Based on the previous lemma and the fact that $f \in W^{1,2}(0, T ; V)$ is absolutely continuous, after possibly being redefined on a set of measure zero, the following estimates can be established by a straightforward computation, see, e.g. [21], [22]. 
Lemma 2.2. For all $n \in N^{*}$

$$
\begin{gathered}
\left\|u_{n}(t)\right\| \leq C_{1}\left(\left\|\lambda_{n}(t)\right\|_{X^{\prime}}+\left\|f_{n}(t)\right\|+\left\|F^{\prime}(0)\right\|\right) \quad \forall t \in[0, T], \\
\left\|u_{n}(t)-\hat{u}_{n}(t)\right\| \leq \frac{T}{n}\left\|\frac{d}{d t} \hat{u}_{n}(t)\right\| \leq C_{2}\left\|f_{n}(t)-f_{n}\left(t-\frac{T}{n}\right)\right\| \\
\leq C_{2} \int_{t-\frac{T}{n}}^{m i n\left\{t+\frac{T}{n}, T\right\}}\|\dot{f}(\tau)\| d \tau \quad \forall t \in[0, T], \\
\left\|\lambda_{n}(t)-\hat{\lambda}_{n}(t)\right\|_{X^{\prime}} \leq \frac{T}{n}\left\|\frac{d}{d t} \hat{\lambda}_{n}(t)\right\|_{X^{\prime}} \\
\leq C_{3}\left\|f_{n}(t)-f_{n}\left(t-\frac{T}{n}\right)\right\| \forall t \in[0, T], \\
\left\|u_{n}-\hat{u}_{n}\right\|_{L^{2}(0, T ; V)}=\frac{T}{n \sqrt{3}}\left\|\frac{d}{d t} \hat{u}_{n}\right\|_{L^{2}(0, T ; V)} \\
\leq C_{2} \frac{T}{n \sqrt{3}}\|\dot{f}\|_{L^{2}(0, T ; V)}, \\
\qquad \hat{\lambda}_{n}\left\|_{L^{2}\left(0, T ; X^{\prime}\right)}=\frac{T}{n \sqrt{3}}\right\| \frac{d}{d t} \hat{\lambda}_{n} \|_{L^{2}\left(0, T ; X^{\prime}\right)} \\
\leq C_{3} \frac{T}{n \sqrt{3}}\|\dot{f}\|_{L^{2}(0, T ; V)} .
\end{gathered}
$$

Using Lemma 2.2, it follows that $\left(\hat{u}_{n}\right)_{n}$ is bounded in $W^{1,2}(0, T ; V),\left(\hat{\lambda}_{n}\right)_{n}$ is bounded in $W^{1,2}\left(0, T ; X^{\prime}\right) \cap L^{\infty}\left(\Xi_{T}\right)$, and since all these functions are absolutely continuous, after possibly being redefined on a set of measure zero, we have the following convergence results.

Lemma 2.3. There exist subsequences of $\left(u_{n}, \hat{u}_{n}\right)_{n}$ and $\left(\lambda_{n}, \hat{\lambda}_{n}\right)_{n}$, denoted by $\left(u_{n_{p}}, \hat{u}_{n_{p}}\right)_{p}$ and $\left(\lambda_{n_{p}}, \hat{\lambda}_{n_{p}}\right)_{p}$, and two elements $u \in W^{1,2}(0, T ; V), \quad \lambda \in$ $W^{1,2}\left(0, T ; X^{\prime}\right) \cap L^{2}\left(\Xi_{T}\right)$ such that

$$
\begin{aligned}
& u_{n_{p}}(t) \rightarrow u(t) \quad \text { in } V \quad \forall t \in[0, T], \\
& \hat{u}_{n_{p}} \rightarrow u \quad \text { in } W^{1,2}(0, T ; V), \\
& \lambda_{n_{p}}(t) \rightarrow \lambda(t) \quad \text { in } \quad X^{\prime} \quad \forall t \in[0, T], \\
& \lambda_{n_{p}}, \hat{\lambda}_{n_{p}} \rightarrow \lambda \text { in } L^{2}\left(0, T ; L^{2}(\Xi)\right), \\
& \hat{\lambda}_{n_{p}} \rightarrow \lambda \text { in } W^{1,2}\left(0, T ; X^{\prime}\right) .
\end{aligned}
$$


Lemma 2.4. For the subsequences $\left(\hat{u}_{n_{p}}\right)_{p},\left(\lambda_{n_{p}}\right)_{p}$, the following relation holds:

$$
\liminf _{p \rightarrow \infty} \int_{0}^{T} \phi\left(\lambda_{n_{p}}(t), \frac{d}{d t} \hat{u}_{n_{p}}(t)\right) d t \geq \int_{0}^{T} \phi\left(\lambda(t), \frac{d}{d t} \hat{u}(t)\right) d t .
$$

Proof. According to Theorem 2.1 with $\mathcal{G}=\left(\hat{\lambda}_{n_{p}}\right)_{p}, \hat{X}=L^{2}(\Xi), \hat{U}=H^{\iota-1 / 2}(\Xi)$, $\hat{Y}=X^{\prime}, p=2,0<\iota<\frac{1}{2}$, and to 47, , 48), we obtain that

$$
\lambda_{n_{p}}, \hat{\lambda}_{n_{p}} \rightarrow \lambda \text { in } L^{2}\left(0, T ; X^{\prime}\right)
$$

By (14) it follows that

$$
\begin{aligned}
& \left|\int_{0}^{T}\left(\phi\left(\lambda_{n_{p}}(t), \frac{d}{d t} \hat{u}_{n_{p}}(t)\right)-\phi\left(\lambda(t), \frac{d}{d t} \hat{u}_{n_{p}}(t)\right)\right) d t\right| \\
& \leq k_{3} \int_{0}^{T}\left\|\lambda_{n_{p}}(t)-\lambda(t)\right\|_{X^{\prime}}\left\|\frac{d}{d t} \hat{u}_{n_{p}}(t)\right\| d t \\
& \leq k_{3}\left\|\lambda_{n_{p}}-\lambda\right\|_{L^{2}\left(0, T ; X^{\prime}\right)}\left\|\frac{d}{d t} \hat{u}_{n_{p}}\right\|_{L^{2}(0, T ; V)},
\end{aligned}
$$

which implies

$$
\lim _{p \rightarrow \infty} \int_{0}^{T}\left(\phi\left(\lambda_{n_{p}}(t), \frac{d}{d t} \hat{u}_{n_{p}}(t)\right)-\phi\left(\lambda(t), \frac{d}{d t} \hat{u}_{n_{p}}(t)\right)\right) d t=0 .
$$

Since by (10), (11), (14), $\phi(\lambda(t), \cdot)$ is convex lower semicontinuous on $V$ for a.e. $t \in[0, T]$, the mapping $\int_{0}^{T} \phi(\lambda(t), \cdot) d t$ is convex lower semicontinuous on $L^{2}(0, T ; V)$ (see, e.g. [23]), so that

$$
\liminf _{p \rightarrow \infty} \int_{0}^{T} \phi\left(\lambda(t), \frac{d}{d t} \hat{u}_{n_{p}}(t)\right) d t \geq \int_{0}^{T} \phi\left(\lambda(t), \frac{d}{d t} \hat{u}(t)\right) d t .
$$

From (51) and (52), 49) follows.

Now, we prove the main strong convergence and existence result.

Theorem 2.3. Under the assumptions (1 - 5), (7) - 16), every convergent subsequence of Lemma 2.3. $\left(u_{n_{p}}, \hat{u}_{n_{p}}\right)_{p},\left(\lambda_{n_{p}}, \lambda_{n_{p}}\right)_{p}$, and their limits $u \in W^{1,2}(0, T ; V), \quad \lambda \in W^{1,2}\left(0, T ; X^{\prime}\right) \cap L^{2}\left(\Xi_{T}\right)$ have the following strong convergence properties

$$
\begin{aligned}
& u_{n_{p}}(t) \rightarrow u(t) \quad \text { in } \quad V \quad \forall t \in[0, T], \\
& \lambda_{n_{p}}(t) \rightarrow \lambda(t) \quad \text { in } \quad X^{\prime} \quad \forall t \in[0, T],
\end{aligned}
$$

and $(u, \lambda)$ is a solution to the problem $Q$. 
Proof. In order to prove (53), we use the same method as the one that enabled to obtain 28. By 10 the sequence $\left(\hat{Q}^{i, n}\right)_{i=0,1, \ldots, n-1}$ implies the following inequality: for every $t \in[0, T]$

$$
\begin{aligned}
&\left\langle F^{\prime}\left(u_{n}(t)\right), v-u_{n}(t)\right\rangle-\left\langle\lambda_{n}(t), l_{0}\left(v-u_{n}(t)\right)\right\rangle_{L^{2}(\Xi)} \\
&+\phi\left(\lambda_{n}(t), v-u_{n}(t)\right) \geq\left\langle f_{n}(t), v-u_{n}(t)\right\rangle \quad \forall v \in V
\end{aligned}
$$

and taking $v=u$, by (6) we derive

$$
\begin{gathered}
F(u(t))-F\left(u_{n_{p}}(t)\right)-\left\langle\lambda_{n_{p}}(t), l_{0}\left(u(t)-u_{n_{p}}(t)\right)\right\rangle_{L^{2}(\Xi)} \\
+\phi\left(\lambda_{n_{p}}(t), u(t)-u_{n_{p}}(t)\right) \geq\left\langle f_{n_{p}}(t), u(t)-u_{n_{p}}(t)\right\rangle+\frac{\alpha}{2}\left\|u(t)-u_{n_{p}}(t)\right\|^{2} \quad \forall p \in \mathbb{N} .
\end{gathered}
$$

Using that $F$ is sequentially weakly lower semicontinuous, (7), (13), the compact embeddings $X \subset L^{2}(\Xi), V \subset U$ and that for all $t \in[0, T]\left(\lambda_{n_{p}}(t)\right)_{p}$ is bounded in $L^{2}(\Xi)$ by $R_{0}$, the previous relation implies

$$
\begin{aligned}
\limsup _{p \rightarrow \infty} & \frac{\alpha}{2}\left\|u(t)-u_{n_{p}}(t)\right\|^{2} \\
\leq & F(u(t))+\limsup _{p \rightarrow \infty}\left(-F\left(u_{n_{p}}(t)\right)\right)+\lim _{p \rightarrow \infty}\left|\left\langle\lambda_{n_{p}}(t), l_{0}\left(u(t)-u_{n_{p}}(t)\right)\right\rangle_{L^{2}(\Xi)}\right| \\
& +\lim _{p \rightarrow \infty} \phi\left(\lambda_{n_{p}}(t), u(t)-u_{n_{p}}(t)\right)-\lim _{p \rightarrow \infty}\left\langle f_{n_{p}}(t), u(t)-u_{n_{p}}(t)\right\rangle \\
& \leq F(u(t))-\liminf _{p \rightarrow \infty} F\left(u_{n_{p}}(t)\right)+\lim _{p \rightarrow \infty}\left\|\lambda_{n_{p}}(t)\right\|_{L^{2}(\Xi)}\left\|l_{0}\left(u(t)-u_{n_{p}}(t)\right)\right\|_{L^{2}(\Xi)} \\
& +\lim _{p \rightarrow \infty} k_{2}\left\|\lambda_{n_{p}}(t)\right\|_{L^{2}(\Xi)}\left\|u(t)-u_{n_{p}}(t)\right\|_{U}-\lim _{p \rightarrow \infty}\left\langle f_{n_{p}}(t), u(t)-u_{n_{p}}(t)\right\rangle \\
& =F(u(t))-\liminf _{p \rightarrow \infty} F\left(u_{n_{p}}(t)\right) \leq 0,
\end{aligned}
$$

which proves (53).

By Theorem 2.1 with $\mathcal{G}=\left(\hat{\lambda}_{n_{p}}\right)_{p}, \hat{X}=L^{2}(\Xi), \hat{U}=H^{\iota-1 / 2}(\Xi), \hat{Y}=X^{\prime}$, $r=2,0<\iota<\frac{1}{2}$, it follows that

$$
\hat{\lambda}_{n_{p}} \rightarrow \lambda \text { in } C\left([0, T] ; X^{\prime}\right),
$$

so that by (41) we obtain (54).

It remains to prove that $(u, \lambda)$ is a solution of the problem $Q$.

First, since $\lambda_{n_{p}}(t) \in \Lambda\left(l\left(u_{n_{p}}(t)\right)\right)$ for all $t \in(0, T)$, we have

$$
\int_{\omega} \underline{\kappa} \circ l\left(u_{n_{p}}\right) \leq \int_{\omega} \lambda_{n_{p}} \leq \int_{\omega} \bar{\kappa} \circ l\left(u_{n_{p}}\right)
$$

for every measurable subset $\omega \subset \Xi_{T}$ and for all $p \in \mathbb{N}$. 
Using (53), (8), the semi-continuity of $\underline{\kappa}$ and $\bar{\kappa}$, the relations (1), (2), 47 , which implies the convergence property $\int_{\omega} \lambda_{n_{p}} \rightarrow \int_{\omega} \lambda$, and passing to limits according to Fatou's lemma, we obtain

$$
\int_{\omega} \underline{\kappa} \circ l(u) \leq \int_{\omega} \lambda \leq \int_{\omega} \bar{\kappa} \circ l(u)
$$

for every measurable subset $\omega \subset \Xi_{T}$, which implies $\lambda(t) \in \Lambda(l(u(t)))$ for almost all $t \in(0, T)$.

Second, integrating both sides in $(21)$ over $[0, T]$ and passing to the limit, by the relations (53), (54), 45), 49, it follows that for all $v \in L^{2}(0, T ; V)$

$$
\begin{aligned}
\int_{0}^{T}\left\langle F^{\prime}(u(t)), v(t)-\dot{u}(t)\right\rangle d t-\int_{0}^{T}\left\langle\lambda(t), l_{0}(v(t)-\dot{u}(t))\right\rangle_{L^{2}(\Xi)} d t \\
\quad+\int_{0}^{T} \phi(\lambda(t), v(t)) d t-\int_{0}^{T} \phi(\lambda(t), \dot{u}(t)) d t \geq \int_{0}^{T}\langle f(t), v(t)-\dot{u}(t)\rangle d t .
\end{aligned}
$$

By Lebesgue's theorem, it follows that $(u, \lambda)$ is a solution of the variational inequality (18).

\section{Applications to two quasistatic contact prob- lems}

Consider an elastic body occupying the set $\Omega \subset \mathbb{R}^{d}, d=2,3$, with $\Gamma=$ $\bar{\Gamma}_{1} \cup \bar{\Gamma}_{2} \cup \bar{\Gamma}_{3}$, where $\Gamma_{1}, \Gamma_{2}, \Gamma_{3}$ are open, disjoint parts of $\Gamma$ and meas $\left(\Gamma_{1}\right)>0$. Assume the small deformation hypothesis and that the inertial effects are negligible.

We denote by $\boldsymbol{u}=\boldsymbol{u}(\boldsymbol{x}, t)$ the displacement field, by $\varepsilon$ the infinitesimal strain tensor and by $\boldsymbol{\sigma}$ the stress tensor, with the components $u=\left(u_{i}\right)$, $\varepsilon=\left(\varepsilon_{i j}\right)$ and $\sigma=\left(\sigma_{i j}\right)$, respectively. We use the classical decompositions $\boldsymbol{u}=u_{N} \boldsymbol{n}+\boldsymbol{u}_{T}, u_{N}=\boldsymbol{u} \cdot \boldsymbol{n}, \boldsymbol{\sigma n}=\sigma_{N} \boldsymbol{n}+\boldsymbol{\sigma}_{T}, \sigma_{N}=(\boldsymbol{\sigma} \boldsymbol{n}) \cdot \boldsymbol{n}$, where $\boldsymbol{n}$ is the outward normal unit vector to $\Gamma$ with the components $n=\left(n_{i}\right)$. The usual summation convention will be used for $i, j, k, l=1, \ldots, d$.

Consider the Hilbert space $\boldsymbol{V}$ and the closed convex sets $L_{-}^{2}\left(\Gamma_{3}\right), \Lambda_{1}(\zeta)$ as follows:

$$
\begin{aligned}
& \boldsymbol{V}=\left\{\boldsymbol{v} \in H^{1}\left(\Omega ; \mathbb{R}^{d}\right) ; \boldsymbol{v}=\mathbf{0} \text { a.e. on } \Gamma_{1}\right\} \\
& L_{-}^{2}\left(\Gamma_{3}\right):=\left\{\delta \in L^{2}\left(\Gamma_{3}\right) ; \delta \leq 0 \text { a.e. in } \Gamma_{3}\right\}, \\
& \Lambda_{1}(\zeta)=\left\{\eta \in L_{-}^{2}\left(\Gamma_{3}\right) ; \underline{\kappa} \circ \zeta \leq \eta \leq \bar{\kappa} \circ \zeta \text { a.e. in } \Gamma_{3}\right\} \quad \forall \zeta \in L^{2}\left(\Gamma_{3}\right) .
\end{aligned}
$$


Assume that in $\Omega$ a body force $\boldsymbol{\varphi}_{1} \in W^{1,2}\left(0, T ; L^{2}\left(\Omega ; \mathbb{R}^{d}\right)\right)$ is prescribed, on $\Gamma_{1}$ the displacement vector equals zero and on $\Gamma_{2}$ a traction $\varphi_{2} \in$ $W^{1,2}\left(0, T ; L^{2}\left(\Gamma_{2} ; \mathbb{R}^{d}\right)\right)$ is applied.

On $\Gamma_{3}$, the contact between the body and a support is possible with the initial gap denoted by $g_{0}$ and the gap corresponding to the solution $\boldsymbol{u}$ denoted by $\left[u_{N}\right]:=u_{N}-g_{0}$. We assume that there exists $\boldsymbol{g} \in \boldsymbol{V}$ such that $g_{N}=g_{0}$ on $\Gamma_{3}$. Since the displacements, their derivatives and the gap are assumed small, we obtain the following unilateral contact condition at time $t:\left[u_{N}\right] \leq 0$ on $\Gamma_{3}$.

On the potential contact surface $\Gamma_{3}$, the displacements and the stress vector will satisfy some contact conditions having the following form:

$$
\underline{\kappa}\left(\left[u_{N}\right]\right) \leq \sigma_{N} \leq \bar{\kappa}\left(\left[u_{N}\right]\right) .
$$

Assume that, for all $\gamma, \delta \in L_{-}^{2}\left(\Gamma_{3}\right)$ and all $\boldsymbol{v}, \boldsymbol{w} \in \boldsymbol{V}$ such that $\gamma \in$ $\Lambda_{1}\left(\left[v_{N}\right]\right), \delta \in \Lambda_{1}\left(\left[w_{N}\right]\right)$,

$$
\left\langle\gamma-\delta, v_{N}-w_{N}\right\rangle_{L^{2}\left(\Gamma_{3}\right)} \leq 0
$$

Different choices for $\underline{\kappa}, \bar{\kappa}$ will give various contact and friction conditions as can be seen in the following examples.

Example 1. (Friction conditions with controlled normal stress)

Let $M \geq 0$ be a constant and define

$$
\underline{\kappa}(s)=\underline{\kappa}_{M}(s)=\left\{\begin{array}{l}
0 \quad \text { if } \quad s<0, \\
-M \quad \text { if } \quad s \geq 0,
\end{array} \quad \bar{\kappa}(s)=\bar{\kappa}_{M}(s)=\left\{\begin{array}{l}
0 \text { if } s \leq 0, \\
-M \quad \text { if } s>0 .
\end{array}\right.\right.
$$

The classical Signorini's conditions correspond formally to $M=+\infty$.

Example 2. (Normal compliance conditions)

Various normal compliance conditions and friction laws can be obtained if one considers $\underline{\kappa}=\bar{\kappa}=\kappa$, where $\kappa: \mathbb{R} \rightarrow \mathbb{R}$ is some negative, decreasing, and bounded Lipschitz continuous function, so that $\sigma_{N}$ is given by the relation $\sigma_{N}=\kappa\left(\left[u_{N}\right]\right)$.

It is easily seen that these two examples verify the condition 600.

Let $\mathcal{F} \geq 0$ be the coefficient of friction, assumed to be a Lipschitz continuous function on $\Gamma$, which ensures to belong to the set of the multipliers on $H^{1 / 2}(\Gamma)$ denoted by $\mathcal{M}$, see, e.g. [2, [4]. Therefore the mapping $H^{1 / 2}(\Gamma) \ni v \mapsto \mathcal{F} v \in H^{1 / 2}(\Gamma)$ is bounded with norm $\|\mathcal{F}\|_{\mathcal{M}}$. 
In order to describe the frictional contact conditions on $\Gamma_{3}$, we define

$$
\begin{gathered}
\forall \boldsymbol{l} \in \boldsymbol{V}, \quad \boldsymbol{S}_{\boldsymbol{l}}:=\left\{\boldsymbol{v} \in \boldsymbol{V} ; \int_{\Omega} \boldsymbol{\sigma}(\boldsymbol{v}) \cdot \boldsymbol{\varepsilon}(\boldsymbol{\psi}) d x=\langle\boldsymbol{l}, \boldsymbol{\psi}\rangle_{\boldsymbol{V}}\right. \\
\left.\forall \boldsymbol{\psi} \in \boldsymbol{V} \text { such that } \boldsymbol{\psi}=\mathbf{0} \text { a.e. on } \Gamma_{3}\right\}, \\
\boldsymbol{L} \in \boldsymbol{V}, \quad\langle\boldsymbol{L}, \boldsymbol{w}\rangle_{\boldsymbol{V}}=\left\langle\boldsymbol{\varphi}_{1}, \boldsymbol{w}\right\rangle_{L^{2}\left(\Omega ; \mathbb{R}^{d}\right)}+\left\langle\boldsymbol{\varphi}_{2}, \boldsymbol{w}\right\rangle_{L^{2}\left(\Gamma_{2} ; \mathbb{R}^{d}\right)} \quad \forall \boldsymbol{w} \in \boldsymbol{V}, \\
\forall \boldsymbol{v} \in \boldsymbol{S}_{\boldsymbol{L}}, \quad\left\langle\sigma_{N}(\boldsymbol{v}), w\right\rangle_{\Gamma}=\int_{\Omega} \boldsymbol{\sigma}(\boldsymbol{v}) \cdot \boldsymbol{\varepsilon}(\overline{\boldsymbol{w}}) d x-\langle\boldsymbol{L}, \overline{\boldsymbol{w}}\rangle_{\boldsymbol{V}} \quad \forall w \in H^{1 / 2}(\Gamma),
\end{gathered}
$$

where $\langle\cdot, \cdot\rangle_{\Gamma}$ denotes the duality pairing on $H^{-1 / 2}(\Gamma) \times H^{1 / 2}(\Gamma), \overline{\boldsymbol{w}} \in \boldsymbol{V}$ satisfies $\overline{\boldsymbol{w}}_{T}=\mathbf{0}$ a.e. on $\Gamma_{3}, \overline{\boldsymbol{w}}_{N}=w$ a.e. on $\Gamma_{3}$. It is easy to verify that for all $\boldsymbol{v} \in \boldsymbol{S}_{\boldsymbol{L}} \sigma_{N}(\boldsymbol{v})$ depends only on the values of $w$ on $\Gamma_{3}$ and not on the choices of $\overline{\boldsymbol{w}}$ having the above properties.

\subsection{A contact problem with Coulomb friction for a nonlinear Hencky material}

Assume that the elastic body satisfies the following nonlinear Hencky-Mises constitutive equation (see [24], 25]):

$$
\boldsymbol{\sigma}(\boldsymbol{u})=\hat{\boldsymbol{\sigma}}(\boldsymbol{u})=\left(k-\frac{2}{3} \mu(\hat{\gamma}(\boldsymbol{u}))\right)(\operatorname{tr} \boldsymbol{\varepsilon}(\boldsymbol{u})) \boldsymbol{I}+2 \mu(\hat{\gamma}(\boldsymbol{u})) \boldsymbol{\varepsilon}(\boldsymbol{u}),
$$

where $k$ is the constant bulk modulus, $\mu$ is a continuously differentiable function in $[0,+\infty)$ satisfying

$$
0<\mu_{0} \leq \mu(r) \leq \frac{3}{2} k, 0<\mu_{1} \leq \mu(r)+2 \frac{\partial \mu(r)}{\partial r} r \leq \mu_{2}, \forall r \geq 0,
$$

and, for all $\boldsymbol{u}, \boldsymbol{v} \in \boldsymbol{V}$,

$\hat{\gamma}(\boldsymbol{u}):=\hat{\gamma}(\boldsymbol{u}, \boldsymbol{u}), \hat{\gamma}(\boldsymbol{u}, \boldsymbol{v})=-\frac{2}{3} \vartheta(\boldsymbol{u}) \vartheta(\boldsymbol{v})+2 \boldsymbol{\varepsilon}(\boldsymbol{u}) \cdot \boldsymbol{\varepsilon}(\boldsymbol{v}), \vartheta(\boldsymbol{u}):=\operatorname{tr} \boldsymbol{\varepsilon}(\boldsymbol{u})=\operatorname{div} \boldsymbol{u}$

Consider the following quasistatic contact problem with Coulomb friction.

Problem $\boldsymbol{P}_{1}^{c}$ : Find $\boldsymbol{u}$ such that $\boldsymbol{u}(0)=\boldsymbol{u}_{0}$ and, for all $t \in(0, T)$,

$$
\begin{aligned}
& \operatorname{div} \boldsymbol{\sigma}(\boldsymbol{u})=-\boldsymbol{\varphi}_{1} \text { in } \Omega, \\
& \boldsymbol{\sigma}(\boldsymbol{u})=\hat{\boldsymbol{\sigma}}(\boldsymbol{u}) \text { in } \Omega, \\
& \boldsymbol{u}=\mathbf{0} \text { on } \Gamma_{1}, \boldsymbol{\sigma} \boldsymbol{n}=\boldsymbol{\varphi}_{2} \text { on } \Gamma_{2}, \\
& \underline{\kappa}\left(\left[u_{N}\right]\right) \leq \sigma_{N} \leq \bar{\kappa}\left(\left[u_{N}\right]\right) \text { on } \Gamma_{3}, \\
& \left|\boldsymbol{\sigma}_{T}\right| \leq \mathcal{F}\left|\sigma_{N}\right| \text { and } \\
& \dot{\boldsymbol{u}}_{T} \neq \mathbf{0} \Rightarrow \boldsymbol{\sigma}_{T}=-\mathcal{F}\left|\sigma_{N}\right| \frac{\dot{\boldsymbol{u}}_{T}}{\left|\dot{\boldsymbol{u}}_{T}\right|} \text { on } \Gamma_{3} .
\end{aligned}
$$


Let $F_{1}: \boldsymbol{V} \rightarrow \mathbb{R}$ be defined by

$$
F_{1}(\boldsymbol{v})=\frac{1}{2} k \int_{\Omega} \vartheta^{2}(\boldsymbol{v}) d x+\frac{1}{2} \int_{\Omega}\left(\int_{0}^{\hat{\gamma}(\boldsymbol{v})} \mu(r) d r\right) d x \quad \forall \boldsymbol{v} \in \boldsymbol{V}
$$

and $J: L_{-}^{2}\left(\Gamma_{3}\right) \times \boldsymbol{V} \rightarrow \mathbb{R}$ be defined by

$$
J(\gamma, \boldsymbol{v})=-\int_{\Gamma_{3}} \mathcal{F} \gamma\left|\boldsymbol{v}_{T}\right| d s \quad \forall \gamma \in L_{-}^{2}\left(\Gamma_{3}\right), \forall \boldsymbol{v} \in \boldsymbol{V} .
$$

One can verify, see, e.g. [24], Ch. 8, that $F_{1}$ is differentiable on $\boldsymbol{V}$ and for all $\boldsymbol{u}, \boldsymbol{v} \in \boldsymbol{V}$

$$
\left\langle F_{1}^{\prime}(\boldsymbol{u}), \boldsymbol{v}\right\rangle_{\boldsymbol{V}}=\int_{\Omega}\left[\left(k-\frac{2}{3} \mu(\hat{\gamma}(\boldsymbol{u}))\right) \vartheta(\boldsymbol{u}) \vartheta(\boldsymbol{v})+2 \mu(\hat{\gamma}(\boldsymbol{u})) \boldsymbol{\varepsilon}(\boldsymbol{u}) \cdot \boldsymbol{\varepsilon}(\boldsymbol{v})\right] d x .
$$

Let $\boldsymbol{u}_{0} \in \boldsymbol{V}, \lambda^{0} \in \Lambda_{1}\left(\left[u_{0 N}\right]\right)$ satisfy the following compatibility condition:

$$
\begin{aligned}
& \left\langle F_{1}^{\prime}\left(\boldsymbol{u}_{0}\right), \boldsymbol{v}-\boldsymbol{u}_{0}\right\rangle_{\boldsymbol{V}}-\left\langle\lambda^{0}, v_{N}-u_{0 N}\right\rangle_{L^{2}\left(\Gamma_{3}\right)} \\
& \quad+J\left(\lambda^{0}, \boldsymbol{v}\right)-J\left(\lambda^{0}, \boldsymbol{u}_{0}\right) \geq\left\langle\boldsymbol{L}(0), \boldsymbol{v}-\boldsymbol{u}_{0}\right\rangle_{\boldsymbol{V}} \quad \forall \boldsymbol{v} \in \boldsymbol{V} .
\end{aligned}
$$

We have the following variational formulation of problem $P_{1}^{c}$.

Problem $\boldsymbol{P}_{1}^{\boldsymbol{v}}:$ Find $\boldsymbol{u} \in W^{1,2}(0, T ; \boldsymbol{V}), \lambda \in W^{1,2}\left(0, T ; H^{-1 / 2}(\Gamma)\right)$ such that $\boldsymbol{u}(0)=\boldsymbol{u}_{0}, \lambda(0)=\lambda^{0}, \lambda(t) \in \Lambda_{1}\left(\left[u_{N}(t)\right]\right)$ for almost all $t \in(0, T)$, and

$$
\begin{aligned}
\left\langle F_{1}^{\prime}(\boldsymbol{u}), \boldsymbol{v}-\dot{\boldsymbol{u}}\right\rangle_{\boldsymbol{V}}-\left\langle\lambda, v_{N}-\dot{u}_{N}\right\rangle_{L^{2}\left(\Gamma_{3}\right)}+J(\lambda, \boldsymbol{v}) & \\
-J(\lambda, \dot{\boldsymbol{u}}) \geq\langle\boldsymbol{L}, \boldsymbol{v}-\dot{\boldsymbol{u}}\rangle_{\boldsymbol{V}} \quad \forall \boldsymbol{v} \in \boldsymbol{V} & \text { a.e. on }(0, T) .
\end{aligned}
$$

The formal equivalence between the variational problem $P_{1}^{v}$ and the classical problem (62)-(66) can be easily proved by using Green's formula. The Lagrange multiplier $\lambda \in L^{2}\left(\Gamma_{3}\right)$ satisfies the relation $\sigma_{N}=\lambda$ in $H^{-1 / 2}(\Gamma)$ that is

$$
\left\langle\sigma_{N}(\boldsymbol{u}), w\right\rangle_{\Gamma}=\langle\lambda, w\rangle_{L^{2}\left(\Gamma_{3}\right)} \quad \forall w \in H^{1 / 2}(\Gamma) .
$$

Taking $\Xi=\Gamma_{3}, \Lambda=\Lambda_{1}, \quad V=\boldsymbol{V}, U=H^{\iota}\left(\Omega ; \mathbb{R}^{d}\right), 1>\iota>\frac{1}{2}, X=$ $H^{1 / 2}(\Gamma), F=F_{1}, \phi=J, f=\boldsymbol{L}$, and $l_{0}(\boldsymbol{v})=v_{N}, l(\boldsymbol{v})=\left[v_{N}\right]=v_{N}-g_{0}$ $\forall \boldsymbol{v} \in \boldsymbol{V}$, it results that the problem $P_{1}^{v}$ is a particular case of problem $Q$.

As it is straightforward to verify the assumptions (1- 5), (7--15), and also (16) if $\|\mathcal{F}\|_{\mathcal{M}}$ is sufficiently small, by Theorem 2.3 we obtain the following existence result.

Proposition 3.1. Under the previous assumptions and if $\|\mathcal{F}\|_{\mathcal{M}}$ is sufficiently small there exists a solution to problem $P_{1}^{v}$. 


\subsection{A contact problem with local friction for a linearly elastic body}

Let $\mathcal{A}$ denote the elasticity tensor, with the components $\mathcal{A}=\left(\mathcal{A}_{i j k l}\right)$ satisfying the following classical symmetry and ellipticity conditions: $\mathcal{A}_{i j k l}=$ $\mathcal{A}_{j i k l}=\mathcal{A}_{k l i j} \in L^{\infty}(\Omega) \forall i, j, k, l=1, \ldots, d, \exists \alpha_{\mathcal{A}}>0$ such that $\mathcal{A}_{i j k l} \tau_{i j} \tau_{k l} \geq$ $\alpha_{\mathcal{A}} \tau_{i j} \tau_{i j} \forall \tau=\left(\tau_{i j}\right)$ satisfying $\tau_{i j}=\tau_{j i}, \forall i, j=1, \ldots, d$.

Consider the following elastic contact problem with Coulomb friction.

Problem $\boldsymbol{P}_{\mathbf{2}}^{c}$ : Find $\boldsymbol{u}$ such that $\boldsymbol{u}(0)=\boldsymbol{u}_{0}$, satisfying

$$
\boldsymbol{\sigma}(\boldsymbol{u})=\mathcal{A} \varepsilon(\boldsymbol{u}) \text { in } \Omega,
$$

and 62 , 64- 66) for all $t \in(0, T)$.

Let us define the bilinear and symmetric mapping $a: \boldsymbol{V} \times \boldsymbol{V} \rightarrow \mathbb{R}$ by

$$
a(\boldsymbol{v}, \boldsymbol{w})=\int_{\Omega} \mathcal{A} \boldsymbol{\varepsilon}(\boldsymbol{v}) \cdot \boldsymbol{\varepsilon}(\boldsymbol{w}) d x=\int_{\Omega} \mathcal{A}_{i j k l} \varepsilon_{i j}(v) \varepsilon_{k l}(w) d x \quad \forall \boldsymbol{v}, \boldsymbol{w} \in \boldsymbol{V}
$$

The form $a$ is continuous on $\boldsymbol{V} \times \boldsymbol{V}$ and, since meas $\left(\Gamma_{1}\right)>0$, by Korn's inequality is also $\boldsymbol{V}$-elliptic.

Let $\boldsymbol{u}_{0} \in \boldsymbol{V}, \lambda^{0} \in \Lambda_{1}\left(\left[u_{0 N}\right]\right)$ satisfy the following compatibility condition:

$$
\begin{aligned}
& a\left(\boldsymbol{u}_{0}, \boldsymbol{v}-\boldsymbol{u}_{0}\right)-\left\langle\lambda^{0}, v_{N}-u_{0 N}\right\rangle_{L^{2}\left(\Gamma_{3}\right)} \\
& +J\left(\lambda^{0}, \boldsymbol{v}\right)-J\left(\lambda^{0}, \boldsymbol{u}_{0}\right) \geq\left\langle\boldsymbol{L}(0), \boldsymbol{v}-\boldsymbol{u}_{0}\right\rangle_{\boldsymbol{V}} \quad \forall \boldsymbol{v} \in \boldsymbol{V} .
\end{aligned}
$$

We have the following variational formulation of problem $P_{2}^{c}$.

Problem $\boldsymbol{P}_{\mathbf{2}}^{\boldsymbol{v}}$ : Find $\boldsymbol{u} \in W^{1,2}(0, T ; \boldsymbol{V}), \lambda \in W^{1,2}\left(0, T ; H^{-1 / 2}(\Gamma)\right)$ such that $\boldsymbol{u}(0)=\boldsymbol{u}_{0}, \lambda(0)=\lambda^{0}, \lambda(t) \in \Lambda_{1}\left(\left[u_{N}(t)\right]\right)$ for almost all $t \in(0, T)$, and

$$
\begin{aligned}
a(\boldsymbol{u}, \boldsymbol{v}-\dot{\boldsymbol{u}})-\left\langle\lambda, v_{N}-\dot{u}_{N}\right\rangle_{L^{2}\left(\Gamma_{3}\right)}+J(\lambda, \boldsymbol{v}) & \\
- & -J(\lambda, \dot{\boldsymbol{u}}) \geq\langle\boldsymbol{L}, \boldsymbol{v}-\dot{\boldsymbol{u}}\rangle_{\boldsymbol{V}} \quad \forall \boldsymbol{v} \in \boldsymbol{V} \quad \text { a.e. on }(0, T) .
\end{aligned}
$$

The Lagrange multiplier $\lambda \in L^{2}\left(\Gamma_{3}\right)$ satisfies again the relation $\sigma_{N}=\lambda$ in $H^{-1 / 2}(\Gamma)$.

Taking $\Xi, \Lambda, V, U, X, \phi, f, l_{0}, l$ as in 3.1 and $F(\boldsymbol{v})=\frac{1}{2} a(\boldsymbol{v}, \boldsymbol{v}) \forall \boldsymbol{v} \in \boldsymbol{V}$, we see that the problem $P_{2}^{v}$ is a particular case of problem $Q$ so that by using again Theorem 2.3 one obtains the following existence result.

Proposition 3.2. Under the previous assumptions and if $\|\mathcal{F}\|_{\mathcal{M}}$ is sufficiently small there exists a solution to problem $P_{2}^{v}$.

Finally, we remark that viscoelastic or viscoplastic bulk behaviors can also be studied by using similar methods to those presented here. 


\section{References}

[1] Telega JJ. Quasistatic Signorini's problem with friction and duality. Int Ser Numer Math. 101. Basel: Birkhäuser Verlag; 1991. p. 199-214.

[2] Andersson LE. Existence results for quasistatic contact problems with Coulomb friction. Appl Math Optim. 2000;42:169-202.

[3] Rocca R, Cocou M. Existence and approximation of a solution to quasistatic Signorini problem with local friction. Int J Engrg Sci. 2001;39:1233-1255.

[4] Rocca R, Cocou M. Numerical analysis of quasistatic unilateral contact problems with local friction. SIAM Journal on Numerical Analysis. 2001;39:1324-1342.

[5] Kikuchi N, Oden JT. Contact problems in elasticity : a study of variational inequalities and finite element methods. SIAM Studies in Applied Mathematics. Philadelphia: SIAM; 1988.

[6] Andersson LE. A quasistatic frictional problem with a normal compliance penalization term. Nonlinear Anal TMA. 1999;37:689-705.

[7] Han W, Sofonea M. Quasistatic contact problems in viscoelasticity and viscoplasticity. Studies in Advanced Mathematics 30. Providence: American Mathematical Society; Somerville: International Press; 2002.

[8] Martins JAC, Oden JT. Existence and uniqueness results for dynamic contact problems with nonlinear normal and friction interface laws. Nonlinear Anal TMA. 1987;11:407-428.

[9] Kuttler KL. Dynamic friction contact problems for general normal and friction laws. Nonlinear Anal TMA. 1997;28:559-575.

[10] Chau O, Han W, Sofonea M. A dynamic frictional contact problem with normal damped response. Acta Applicandae Mathematicae. 2002;71:159-178.

[11] Migórski S, Ochal A, Sofonea M. Nonlinear inclusions and hemivariational inequalities. dvances in Mechanics and Mathematics 26. New York: Springer; 2013.

[12] Shillor M, Sofonea M, Telega JJ. Models and analysis of quasistatic contact. Lect Notes Phys 655. Berlin, Heidelberg: Springer; 2004. 
[13] Badea L, Cocou M. Internal and subspace correction approximations of implicit variational inequalities. Mathematics and Mechanics of Solids. 2015;20:1026-1048.

[14] Capatina A. Variational inequalities and frictional contact problems. Advances in Mechanics and Mathematics 31. New York: Springer; 2014.

[15] Rabier PJ, Savin OV. Fixed points of multi-valued maps and static Coulomb friction problems. J. Elasticity. 2000;58:155-176.

[16] Cocou M. A class of dynamic contact problems with Coulomb friction in viscoelasticity. Nonlinear Anal RWA. 2015;22:508-519.

[17] Cocou M. A variational analysis of a class of dynamic problems with slip dependent friction. Annals of the University of Bucharest (mathematical series). 2014;5(LXIII):279-297.

[18] Simon J. Compact sets in the space $L^{p}(0, T ; B)$. Ann Mat Pura Appl. 1987;146:65-96.

[19] Fan K. Fixed points and minimax theorems in locally convex topological linear spaces. Proc Nat Acad Sci USA. 1952;38:121-126.

[20] Browder F. Nonlinear operators and nonlinear equations of eyolution in Banach spaces. Proceedings of Symposia in Pure Mathematics, Vol. 18, Part 2. Providence: American Mathematical Society; 1976.

[21] Cocou M, Pratt E, Raous M. Formulation and approximation of quasistatic frictional contact. Int J Engng Sci. 1996;34:783-798.

[22] Capatina A, Cocou M, Raous M. A class of implicit variational inequalities and applications to frictional contact. Math Methods Appl Sci. 2009;32:1804-1827.

[23] Brezis H. Problèmes unilatéraux. Journal de Mathématiques Pures et Appliquées. 1972;51:1-168.

[24] Nečas J, Hlaváček I. Mathematical theory of elastic and elasto-plastic bodies. An introduction. Studies in Appl Math 3. Amsterdam: Elsevier; 1981.

[25] Zeidler E. Nonlinear functional analysis and its applications. Vol. IV: Applications to mathematical physics. New York: Springer-Verlag; 1997. 